\title{
Electric field dependence of the electron mobility in bulk wurtzite $\mathrm{ZnO}$
}

\author{
K ALFARAMAWI \\ Science Department, Teachers College, King Saud University, Riyadh 11451, Saudi Arabia \\ Physics Department, Faculty of Science, Alexandria University, Alexandria 21511, Egypt
}

MS received 7 November 2013; revised 1 January 2014

\begin{abstract}
The electric field dependence of the electron mobility in bulk wurtzite zinc oxide ( $\mathrm{ZnO})$ material is studied. The low-field electron mobility is calculated as a function of doping concentration and lattice temperature. The results show that above nearly $50 \mathrm{~K}$ the electrical conduction is governed by activation through the bulk material and the conduction is then influenced by both lattice and impurity scattering mechanisms. The high-field characteristics are also considered. The transition between the low-field and high-field regions is specified. The negative differential mobility for bulk $\mathrm{ZnO}$ at room temperature is observed at electric fields above $280 \mathrm{kV} / \mathrm{cm}$.
\end{abstract}

Keywords. Electron drift velocity; low-field mobility; saturation velocity.

\section{Introduction}

Recently, zinc oxide $(\mathrm{ZnO})$ semiconductor has received a great attention for electronic and optoelectronic applications because of its noticeable advantages over $\mathrm{GaN}$ and other IIInitrides. $\mathrm{ZnO}$ has unique properties with possible application to ultraviolet light emitters, gas sensors, surface acoustic wave devices and transparent electronics (Ozgur et al 2005).

A distinguished characteristic of $\mathrm{ZnO}$, compared with many other direct gap semiconductors, is the large intervalley separation which is approximately $3.23 \mathrm{eV}$, while this is $330 \mathrm{meV}$ in GaAs and $2.25 \mathrm{eV}$ in $\mathrm{GaN}$. This may lead to a use of a simple analytical nonparabolic approximation for the conduction band around the centre of the Brillouin zone even at moderately high electric field up to several hundred $\mathrm{kV} / \mathrm{cm}$ (Goano et al 2007).

The electron mobility in bulk $\mathrm{ZnO}$ as a function of electric field is important parameter for device design and analysis. At low-field level, the bulk sample carriers are almost in equilibrium with the lattice vibrations and the low-field mobility is mainly affected by phonon and Coulomb scattering (Schroder 1987). At higher electric fields, mobility becomes field-dependent parameter.

There are many theoretical models to describe the lowfield mobility for bulk materials. Those include the constant mobility model, Caughey and Thomas (1967) model and Arora model (Arora et al 1982). In such models, the low-field mobility is temperature- and doping-concentrationdependent. On the other hand, Dorkel and Letureq (1981) presented a model, where the dependence on the carriercarrier scattering was considered in the low-field mobility formulation. Klaassen (1992) model provided unified description of majority and minority carrier mobility, where

$\overline{\text { (kalgarmawy@ksu.edu.sa) }}$ the effects of lattice scattering, screened Coulomb charges, carrier-carrier scattering and impurity clustering effects at high concentrations are considered.

In this paper, the electric-field dependent electron mobility in bulk wurtzite $\mathrm{ZnO}$ is studied. The low-field electron mobility, as a function of doping concentration and lattice temperature, is computed. High-field electron mobility and the electron saturation velocity are also studied. The transition between the low-field and high-field regions is determined.

\section{Theory}

The low-field mobility models are best understood by starting with the modelling approach of Caughey and Thomas (1967),

$$
\mu_{0}=\mu_{\min }+\frac{\mu_{\max }-\mu_{\min }}{1+(N / C)^{\alpha}}
$$

where $C$ and $\alpha$ are fitting parameters, $N$ the total doping density and $\mu_{\max }$ and $\mu_{\min }$ the 'maximum-minimum' behaviour of the mobility.

To match the experimental findings, at doping levels greater than $5 \times 10^{19} \mathrm{~cm}^{-3}$, Masetti et al (1983) assumed that:

$$
\mu_{0}=\mu_{\min }+\frac{\mu_{\max }-\mu_{\min }}{1+\left(N / C_{1}\right)^{\alpha_{1}}}-\frac{\mu_{1}}{1+\left(C_{2} / N\right)^{\alpha_{2}}},
$$

where $\mu_{1}, C_{1}, C_{2}, \alpha_{1}$ and $\alpha_{2}$ are new fitting parameters.

According to Arora (Arora et al 1982), the temperature $(T)$ and ionized impurity concentration $\left(N_{\mathrm{i}}\right)$ dependence of the low-field mobility is given by:

$$
\mu_{0}=\mu_{\mathrm{om}}+\frac{\mu_{\mathrm{d}}}{1+\left(N_{\mathrm{i}} / N_{0}\right)^{\mathrm{r}}},
$$


where

$$
\begin{aligned}
\mu_{\mathrm{om}} & =r_{\mathrm{m}}\left(\frac{T}{T_{0}}\right)^{\alpha_{\mathrm{m}}}, & \mu_{\mathrm{d}}=r_{\mathrm{d}}\left(\frac{T}{T_{0}}\right)^{\alpha_{\mathrm{d}}}, \\
N_{0} & =r_{\mathrm{N}}\left(\frac{T}{T_{0}}\right)^{\alpha_{\mathrm{N}}}, & r=r_{\mathrm{a}}\left(\frac{T}{T_{0}}\right)^{\alpha_{\mathrm{a}}},
\end{aligned}
$$

with $T_{0}=300 \mathrm{~K}$ and the quantities $r_{\mathrm{m}}, r_{\mathrm{d}}, r_{\mathrm{N}}, r_{\mathrm{a}}, \alpha_{\mathrm{m}}, \alpha_{\mathrm{d}}$, $\alpha_{\mathrm{N}}$ and $\alpha_{\mathrm{a}}$ are fitting parameters.

The high-field electron characteristics are well understood by studying the relationship between the drift velocity $v(E)$ and the electric field. By the aid of Arora model, one can write

$$
v(E)=\frac{\mu_{0} E+v_{\mathrm{sat}}\left(E / E_{\mathrm{c}}\right)^{\beta_{1}}}{1+\left(E / E_{\mathrm{c}}\right)^{\beta_{1}}+a\left(E / E_{\mathrm{c}}\right)^{\beta_{2}}},
$$

where $\mu_{0}$ is the temperature- and doping-dependent low-field electron mobility given from (3), $v_{\text {sat }}$ the saturation velocity, $E_{\mathrm{c}}$ the critical field corresponding to the maximum steady state velocity and $a, \beta_{1}, \beta_{2}$ the fitting parameters.

The temperature dependence of the drift velocity is properly specified by considering the model which suggested that the fitting parameters could be modified to be temperaturedependent according to the equation (Yang et al 2010)

$$
\operatorname{par}(T)=\operatorname{par}(300) \times\left[a+b T+c T^{2}\right],
$$

where $\operatorname{par}(300)$ is a parameter at $T=300 \mathrm{~K}$ and $a, b$ and $c$ are constants.

\section{Results and discussion}

Calculations of the low-field electron mobility and highfield electron characteristics as a function of temperature and doping concentration for bulk wurtzite $\mathrm{ZnO}$ were carried out according to (3) and (4). The numerical values of the parameters used in the calculations are given in table 1.

Table 1. Parameters used in the fielddependent electron mobility calculations using (3) and (4) for bulk wurtzite $\mathrm{ZnO}$ (extracted from Furno et al 2008).

\begin{tabular}{ll}
\hline Parameter & \multicolumn{1}{c}{ Value } \\
\hline$r_{\mathrm{m}}$ & $98.92 \mathrm{~cm}^{2} / \mathrm{V} \cdot \mathrm{s}$ \\
$r_{\mathrm{d}}$ & $304 \cdot 1 \mathrm{~cm}^{2} / \mathrm{V} \cdot \mathrm{s}$ \\
$r_{\mathrm{N}}$ & $1.92 \times 10^{17} \mathrm{~cm}^{-3}$ \\
$r_{\mathrm{a}}$ & 1.058 \\
$\alpha_{\mathrm{m}}$ & -0.698 \\
$\alpha_{\mathrm{d}}$ & -3.386 \\
$\alpha_{\mathrm{N}}$ & 2.718 \\
$\alpha_{\mathrm{a}}$ & $-0 \cdot 125$ \\
$E_{\mathrm{c}}$ & $290 \mathrm{kV} / \mathrm{cm}$ \\
$a$ & 2.4 \\
$\beta_{1}$ & 4.5 \\
$\beta_{2}$ & 0.85 \\
\hline
\end{tabular}

Figure 1 shows the low-field electron mobility vs temperature for $\mathrm{ZnO}$ in a temperature range from 10 to $400 \mathrm{~K}$. The mobility was calculated at different values of donor concentration from $3 \times 10^{16}$ up to $5 \times 10^{17} \mathrm{~cm}^{-3}$. In figure 1, two main regions can be distinguished. In temperature range, between 10 and approximately $50 \mathrm{~K}$ (the value depends on the carrier concentration), the electron mobility increases with the increase of temperature. The second range is from 50 up to $400 \mathrm{~K}$ in which the mobility decreases with the elevation of temperature.

Above approximately $50 \mathrm{~K}$, the conduction is assumed to be dependent on the activation processes of electrons between donor sites and the conduction band. In that range of temperature, scattering of electrons determines the mobility behaviour. Scattering by acoustic and optical phonons are probably the dominant processes at higher temperatures, while at lower temperatures, the ionized impurity scattering process might be the dominant one.

Below $50 \mathrm{~K}$, the mobility data are assumed to be determined by both band and hopping conduction (Look et al 1998). The experimental data of low-field mobility on bulk wurtzite $\mathrm{ZnO}$ at carrier concentration $6 \times 10^{16} \mathrm{~cm}^{-3}$ shows that the critical temperature between the two types of conduction is about $40 \mathrm{~K}$, which may be considered in a good agreement with our calculations (Bertazzi et al 2009).

In order to study the temperature-dependent electronic behaviour, the low-field mobility is plotted as a function of $T$ for temperatures above $100 \mathrm{~K}$ at some selected values of the donor concentration, see figure 2. Experimental Hall mobility data extracted from Bellotti and Bertazzi (2007) are also included as well as results from Monte Carlo simulation (ABMC) data from Furno et al (2008). Inspection of figure 2 reveals that the present calculated results fit well with the experimental results of Bellotti and Bertazzi (2007).

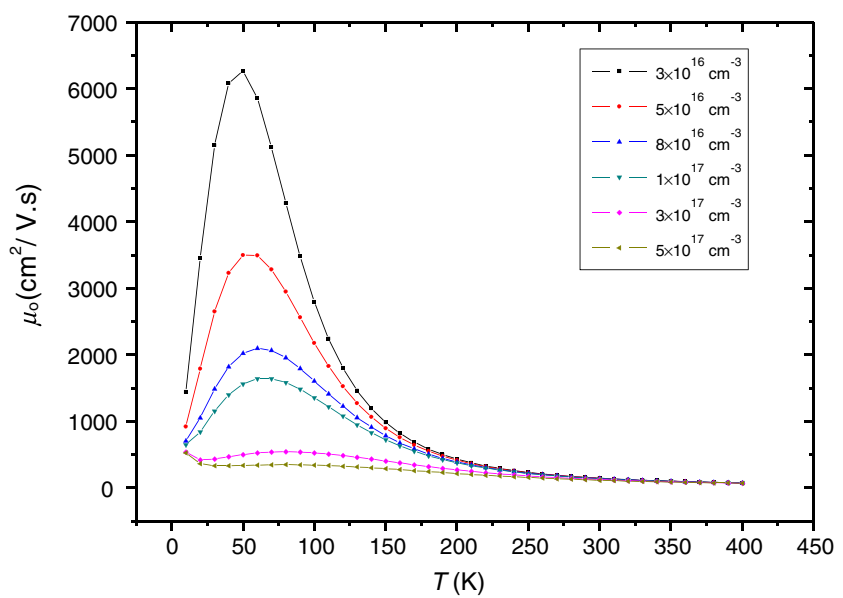

Figure 1. Low-field electron mobility vs temperature for $\mathrm{ZnO}$ in a temperature range from $10 \mathrm{up}$ to $400 \mathrm{~K}$. The curves are calculated at different values of donor concentration from $3 \times 10^{16}$ up to $5 \times 10^{17} \mathrm{~cm}^{-3}$. Peak mobilities are noticed at approximately $50 \mathrm{~K}$ (the value depends on the carrier concentration). 


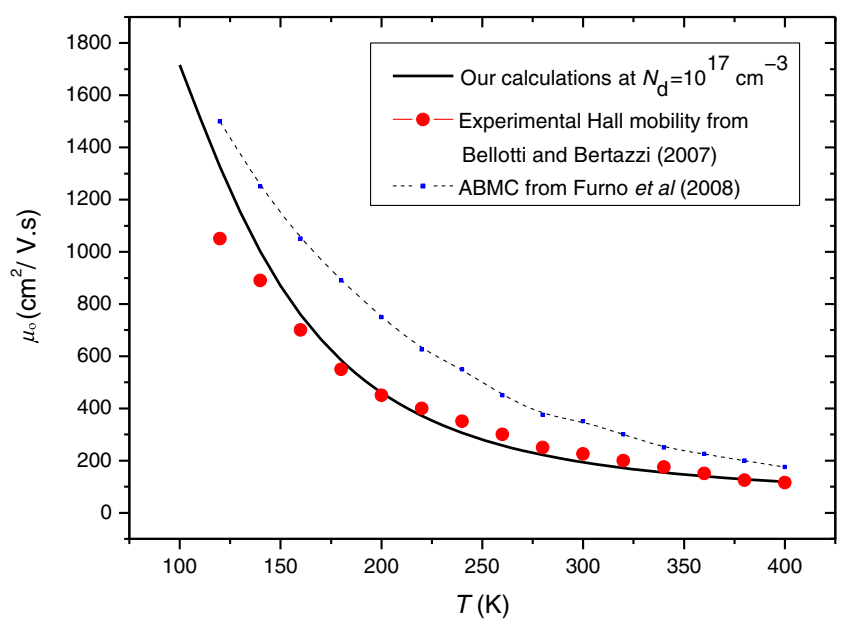

Figure 2. Low-field electron mobility at temperatures above $100 \mathrm{~K}$ for bulk wurtzite $\mathrm{ZnO}$ calculated at donor concentration of $10^{17} \mathrm{~cm}^{-3}$ (solid line). The calculated results are compared to experimental work from Bellotti and Bertazzi (2007) (symbols) and Monte Carlo simulation from Furno et al (2008) (dashed line).

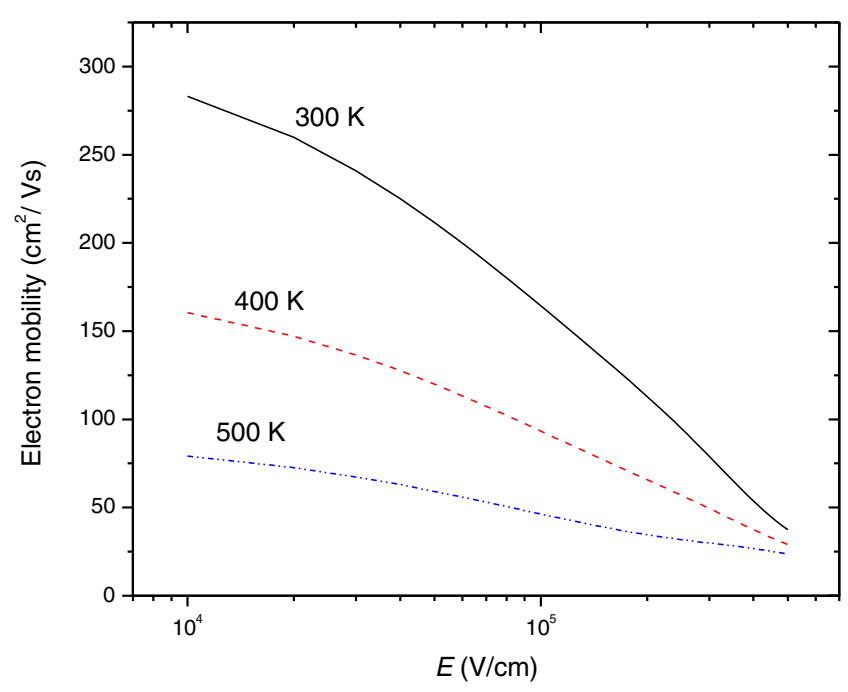

Figure 3. Electron mobility vs electric field at temperatures 300, 400 and $500 \mathrm{~K}$ for bulk wurtzite $\mathrm{ZnO}$.

The room temperature $(300 \mathrm{~K})$ electron mobility extracted from figure 1 was $200 \mathrm{~cm}^{2} / \mathrm{V} \cdot \mathrm{s}$. This result is close to the value of $205 \mathrm{~cm}^{2} / \mathrm{V} \cdot \mathrm{s}$ which is reported by Bellotti and Bertazzi (2007) and Furno et al (2008).

The electron mobility of bulk $\mathrm{ZnO}$ was investigated with the variation of the electric field strength up to $500 \mathrm{kV} / \mathrm{cm}$. Figure 3 represents the change of the electron mobility vs the electric field at different temperatures: 300, 400 and $600 \mathrm{~K}$. It is evident from figure 3 that the mobility is field-dependent and it decreases with increase in the electric field. This is probably due to increased lattice scattering at higher carrier energies gained by the field.

The high-field electron mobility characteristics can be understood by determining the electron drift velocity, $v(E)$

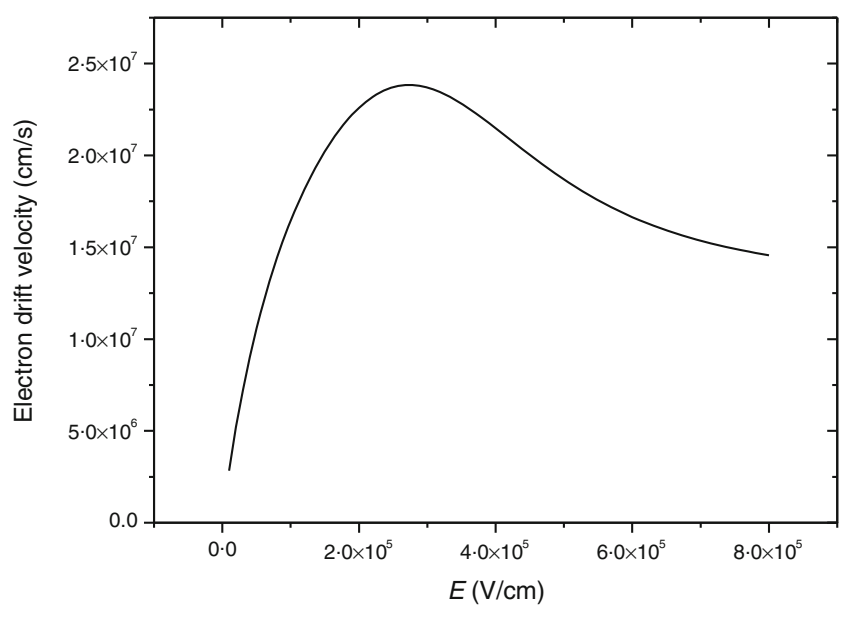

Figure 4. Electron drift velocity vs electric field at room temperature for bulk wurtzite $\mathrm{ZnO}$.

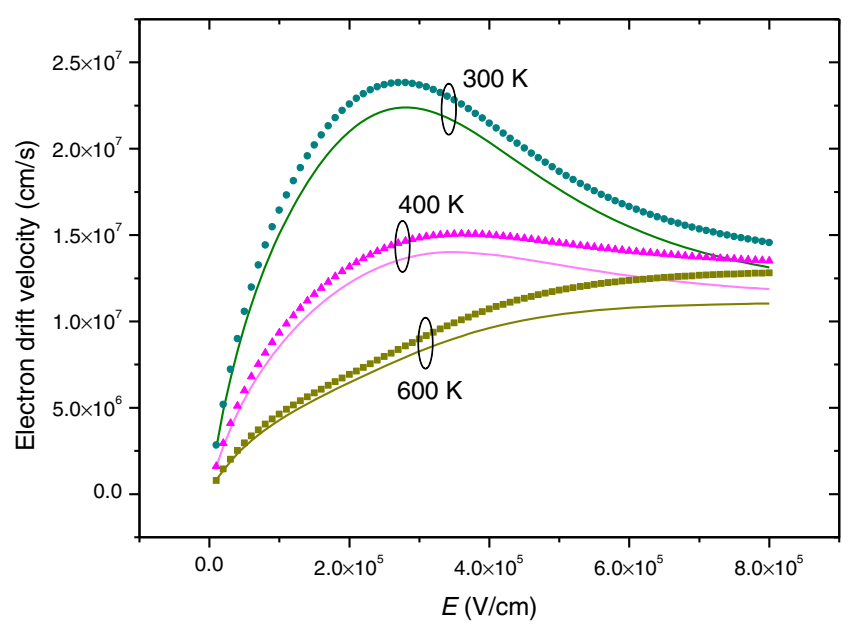

Figure 5. Electron drift velocity vs electric field at 300, 400 and $600 \mathrm{~K}$ for bulk wurtzite $\mathrm{ZnO}$. The lines represent our calculations using Yang et al (2010) model and symbols represent calculations using Arora et al (1982) model.

as a function of the electric field. Equation (5) is used to calculate the drift velocity with the variant electric field. Figure 4 shows the dependence of the drift velocity on the electric field at room temperature. The dependence of $v(E)$ on the electric field presents a dual-slope behaviour, similar to that observed in GaN and other III-nitrides (Bellotti et al 2001). Inspection of figure 4 reveals that there is a negative differential mobility (NDM) at room temperature observed at electric fields above $280 \mathrm{kV} / \mathrm{cm}$. This value can be considered as a distinguished point between the low- and the high-field characteristics.

In order to examine the model suggested that the parameters are all temperature-dependent (6) as introduced by Yang et al (2010). Figure 5 demonstrates the electron drift velocity calculated using this model compared to that calculated from (5). The numerical values used in the 
Table 2. Parameters used in the temperature-dependent high field characteristics calculations using the Yang model for bulk wurtzite $\mathrm{ZnO}$ (Yang et al 2010).

\begin{tabular}{lccc}
\hline Parameters & $a$ & $b$ & $c$ \\
\hline$E_{\mathrm{c}}$ & 0.521 & $1.944 \times 10^{-3}$ & $-1.175 \times 10^{-6}$ \\
$v_{\text {sat }}$ & 1.581 & $-2.431 \times 10^{-3}$ & $1.35 \times 10^{-6}$ \\
$a$ & 3.086 & $-9.823 \times 10^{-3}$ & $9.608 \times 10^{-6}$ \\
$\beta_{1}$ & 0.9611 & $6.297 \times 10^{-3}$ & $-2.358 \times 10^{-7}$ \\
$\beta_{2}$ & 0.6687 & $1.223 \times 10^{-3}$ & $-3.475 \times 10^{-7}$ \\
\hline
\end{tabular}

calculations are listed in table 2. In the figure, the lines show the calculations using Yang model and the symbols are due to Arora model (5). The same trends can be observed.

At high electric fields, the electron drift velocity decreases due to increased intra- and inter-valley scattering. It is reported that the electron population of $\mathrm{ZnO}$ in the $\Gamma$-valley is high (Arabshahi et al 2009). Then, the velocity is influenced by scattering processes at high electric fields. The effect of the field then becomes more dominant than the effect of temperature and consequently, it becomes temperature independent.

\section{Conclusions}

The low-field electron mobility is carried out as a function of temperature from 10 to $400 \mathrm{~K}$ at different doping concentrations. The results show that above nearly $50 \mathrm{~K}$, the conduction is influenced by both lattice and impurity scattering mechanisms. The dependence of the electron mobility on the electric field shows a maximum electron drift velocity at electric field around $280 \mathrm{kV} / \mathrm{cm}$. This probably determines the distinct value between low-field and high-field regions and then the negative differential mobility is observed at electric fields above $280 \mathrm{kV} / \mathrm{cm}$.

\section{Acknowledgements}

The author wishes to express his appreciation to the Deanship of Scientific Research at King Saud University, Saudi Arabia, for funding this work. The author also thanks Prof S Abboudy and Prof L Abulnasr for their valuable comments and suggestions.

\section{References}

Arabshahi H, Rokn-Abadi R and Golafroz S 2009 Modern Phys. Lett. B23 2807

Arora N D, Hauser J R and Roulston D J 1982 IEEE Trans. Electron. Dev. 29292

Bellotti E et al 2001 Int. J. High Speed Electron. Syst. 11525

Bellotti E and Bertazzi F 2007 Transport parameters for electrons and holes In J Piprek (ed.) Nitride semiconductor devices: Principles and simulation (Weinheim: Wiley-VCH Verlag) Chap. 4, p 69

Bertazzi F, Bellotti E, Furno E and Goano M 2009 J. Electron. Mater. 381677

Caughey D M and Thomas R E 1967 Proc. IEEE 552192

Dorkel J M and Leturcq P H 1981 Solid-State Electron. 248211

Furno E, Bertazzi F, Goano M, Ghione G and Bellotti E 2008 Solid State Electron. 521796

Goano M, Bertazzi F, Penna M and Bellotti E 2007 J. Appl. Phys. 102083709

Klaassen D B M 1992 Solid-State Electron. 35953

Look D C, Reynolds D C, Sizelove J R, Jones R L, Litton C W, Cantwell G and Harsch W C 1998 Solid State Commun. 105339

Masetti G, Severi M and Solmi S 1983 IEEE Trans. Electron Dev. 30764

Ozgur U, Alivov Y I, Liu C, Teke A, Reshchikov M A, Dogan S, Avrutin V, Cho S J and Morkoc H 2005 J. Appl. Phys. 98041301

Schroder D K 1987 Modular Series on solid state devices: Advanced MOS Devices (New York: Addison-Wesley Publishing Company)

Yang L, Yang Yao Q, Zhang X, Liu Q and Hao V 2010 Proceedings of 10th IEEE International Conference on Solid-State and Integrated Circuit Technology (ICSICT) (Shanghai, China: IEEE Press) p 1566 